This is a transaction of the proceedings of The 70th Annual Meeting of The Society of Materials Science, Japan as "Development of Novel Construction Material from Food Waste".

\title{
Development of Novel Construction Material from Food Waste
}

\author{
Kota MACHIDA ${ }^{1}$ and Yuya SAKAI ${ }^{*}$ \\ ${ }^{1}$ School of Engineering, The University of Tokyo, 4-6-1 Komaba, Meguro, Tokyo 153-8505, Japan. \\ ${ }^{2}$ Institute of Industrial Science, The University of Tokyo, 4-6-1 Komaba, Meguro, Tokyo 153-8505, \\ Japan. \\ *Corresponding author, E-mail: ysakai@iis.u-tokyo.ac.jp
}

\section{Highlights}

- A new material with a bending strength higher than that of concrete was developed using vegetable or fruit waste

- The new material maintains the color, taste, and flavor of the original vegetable or fruit

- Without water resistant treatment, the material is edible and can be conditioned with seasonings

\section{Introduction}

The reduction of food waste has become a growing concern recently, and the significant amount of waste generated from food is not sufficiently recycled. In Japan, uneaten food is segregated into two types: food loss and food waste. Food loss refers to waste that is considered still edible, consisting of leftovers, from the food industry or homes, that are not eaten. Food waste consists of non-edible parts of foods such as peels, seeds of vegetables and fruits, and bones. In 2014, approximately 6 million tons of the former and 21 million tons of the latter were generated in Japan ${ }^{1}$. Although $60 \%$ was recycled into feeding for livestock or composted and the remaining waste was burnt, the profitability and efficiency of these methods are not sufficient for sustainable recycling.

Considering the above background, this research aimed to develop new materials from food waste, with sufficient bending strength for practical use. As these materials were made with edible raw materials, such as peels of vegetables or fruits, factors related to edibility, including flavor, texture, surface color, and others, were quantitatively evaluated. Seaweed was also used as a raw material in the experiment, as mass generation of seaweed is considered a significant environmental issue. The study aimed to produce specimens with a bending strength greater than $5 \mathrm{MPa}$, which corresponds to that of ordinary concrete, and an acceptable edibility.

\section{Methods}

A technique to produce construction materials from wood powder by heat pressing was previously 
This is a transaction of the proceedings of The 70th Annual Meeting of The Society of Materials Science, Japan as "Development of Novel Construction Material from Food Waste".

developed; in this technique, wood powder is used as a cohesive material ${ }^{1}$. Heat pressing of dried powder made from food waste or seaweed was conducted based on this technique in the current study.

\subsection{Preparation and production}

The following materials were used for the experiments: peels of oranges, onions, pumpkins, and bananas; the outer leaves of Chinese and other cabbages; and seaweed. To improve the taste and for reinforcement, salt, sugar, and natural edible clay were added. After cutting the raw materials into small pieces, they were placed in an oven set at a temperature of $105^{\circ} \mathrm{C}$ or a vacuum drying machine for drying.

The dried materials were pulverized using a blender or disk mill. The powder was mixed with water and seasonings and heat pressed under the respective forming conditions ranging 6-50 MPa and 60$180{ }^{\circ} \mathrm{C}$. The measured experimental conditions included the water content in weight, forming temperature, pressure, and duration.

\subsection{Evaluation}

A three-point bending test was conducted to evaluate bending strength. Flavor, smell, and appearance were evaluated through tasting and observation by the authors. To investigate the effect of aging, some specimens were cut into two pieces: one was exposed to the air without any cover, and the other was sealed in an air-tight container. All pieces were kept in a room of $23{ }^{\circ} \mathrm{C}$. After four months, observations were conducted to compare the differences in smell and appearance between the two cases.

\section{Results}

\subsection{Formation}

Two types of failure tendencies were observed. When the forming temperature or the water content was too high, the materials melted and flowed out of the mold, as shown on the left-hand side of Fig. 1. When the forming temperature was too low, the specimens became fragile and broke easily when taken out of the mold, as shown in the right side of Fig. 1. All the raw materials used in this study were successfully formed in construction materials by controlling the forming conditions as introduced in Section 3.3. Figure 2 shows a specimen successfully formed from seaweed powder.

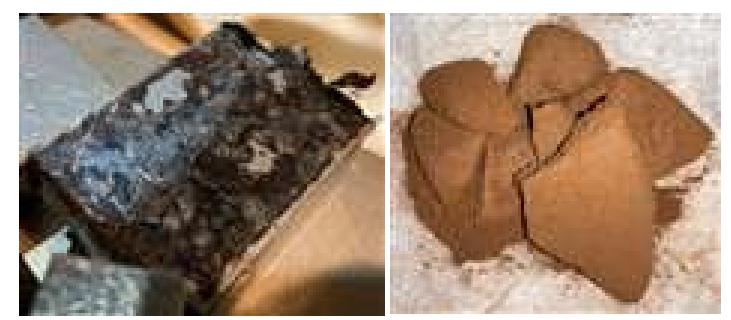

Fig. 1 Samples that failed to form. 
This is a transaction of the proceedings of The 70th Annual Meeting of The Society of Materials Science, Japan as "Development of Novel Construction Material from Food Waste".

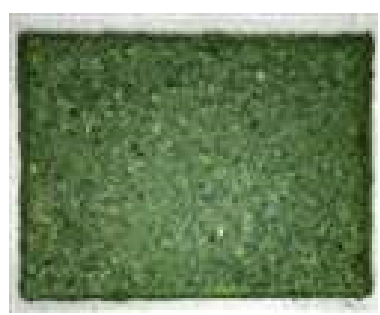

Fig. 2 A sample made from seaweed.

\subsection{Comparison of the drying methods}

Differences in appearance and flavor were observed between the two drying methods (Fig. 3). Peels of oranges and onions were used for the comparison. Burns were observed on the surface of the specimens formed from oven-dried materials, and the original color of the oranges and onions was lost. Their taste was bitter. Specimens formed from vacuum-dried oranges and onions retained their original colors, flavors, and smells.

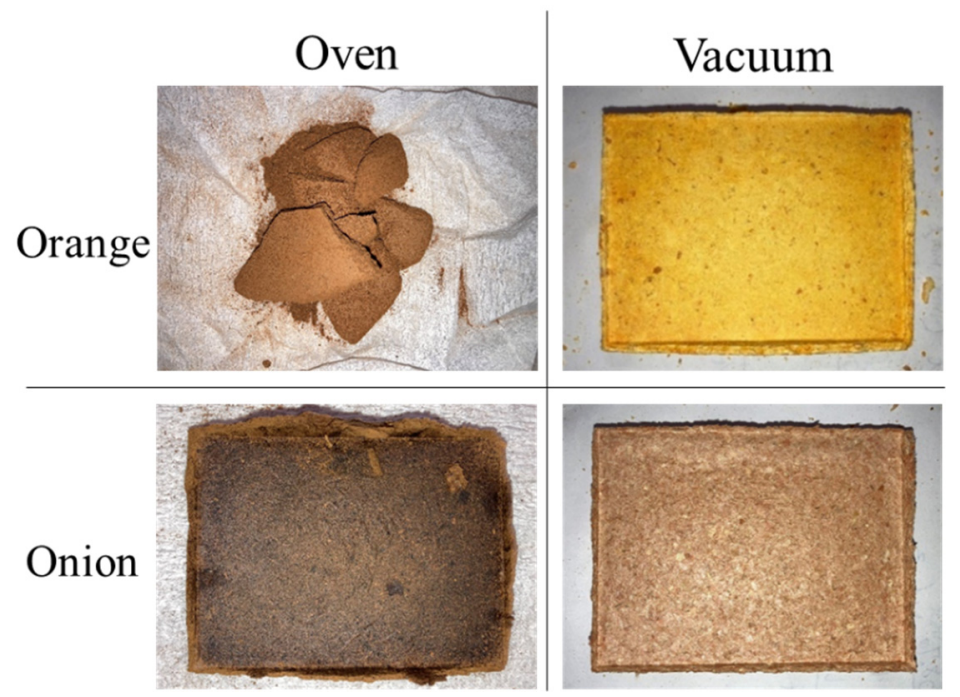

Fig. 3 Comparison of the drying methods.

\subsection{Different bending strengths of materials}

The basic forming conditions, as shown in Table 1, were defined through preliminary experiments. The forming temperature of the seaweed was $120^{\circ} \mathrm{C}$. Figure 4 shows the bending strength of the specimens formed under these conditions. All materials except pumpkin satisfied a bending strength of $5 \mathrm{MPa}$. In particular, the bending strength of Chinese cabbage was $17.7 \mathrm{MPa}$, which is three times higher than the bending strength of ordinary concrete. In the figure, a mixture of pumpkin, which showed the lowest strength, and Chinese cabbage, which showed the highest strength, is also shown. A quarter of the pumpkin powder by weight was replaced with Chinese cabbage, and the resulting material showed an improved strength of approximately $10 \mathrm{MPa}$. This result indicates that weaker 
This is a transaction of the proceedings of The 70th Annual Meeting of The Society of Materials Science, Japan as "Development of Novel Construction Material from Food Waste".

food materials can be strengthened by adding stronger ones.

Table 1. Heat-pressing conditions

\begin{tabular}{cccc}
\hline Temperature & $\begin{array}{c}\text { Water } \\
\text { content }\end{array}$ & Pressure & Duration \\
\hline $100^{\circ} \mathrm{C}$ & $0 \%$ & $50 \mathrm{MPa}$ & $10 \mathrm{~min}$ \\
\hline
\end{tabular}

*seaweed: $120^{\circ} \mathrm{C}$

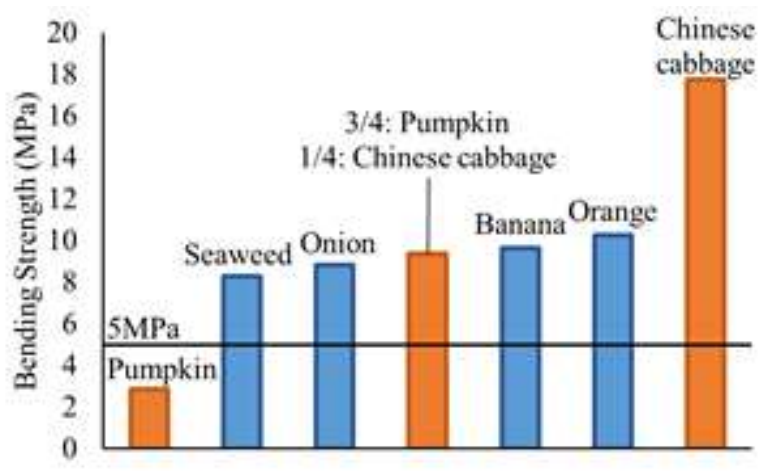

Fig. 4 Bending strength

\subsection{Addition of seasonings}

To improve taste, sugar or salt was added to the orange peel powder. Figure 5 shows the bending strength when a quarter of the weight of the powder is replaced by sugar or salt. Both the seasonings satisfied a bending strength of $5 \mathrm{MPa}$. Furthermore, salt increased the bending strength. The addition of seasonings reduced the bitterness of the peels and improved the flavor. The mechanism of the increase in strength by seasoning is not clear and needs to be investigated in the future.

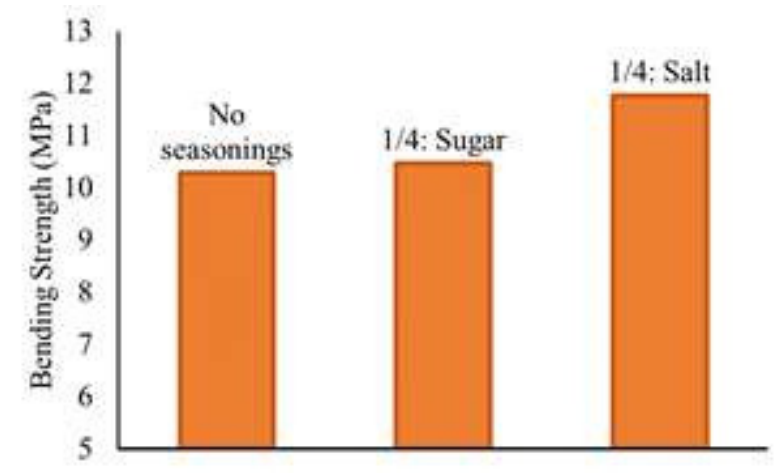

Fig. 5 Effect of seasonings on bending strength.

\subsection{Addition of natural edible clay}

Natural edible clay was added to the pulverized peels of oranges and bananas and the outside leaves 
This is a transaction of the proceedings of The 70th Annual Meeting of The Society of Materials Science, Japan as "Development of Novel Construction Material from Food Waste".

of the cabbages in three replacement proportions by weight for comparison. Figure 6 shows the bending strength comparison. When orange peel was replaced by clay, the material strength reduced compared to that without clay, regardless of the replacement rate. In contrast, when a quarter of the cabbage or banana peels by weight was replaced with clay, the material bending strength increased compared to the original strengths. As the replacement proportion increased, the surface color of the specimens changed to gray from their original color, as shown in Fig. 7. There was also a small effect on flavor, and the texture became similar to that of flour. The mechanism of the increase in strength by the addition of clay is not clear and needs to be investigated in the future..

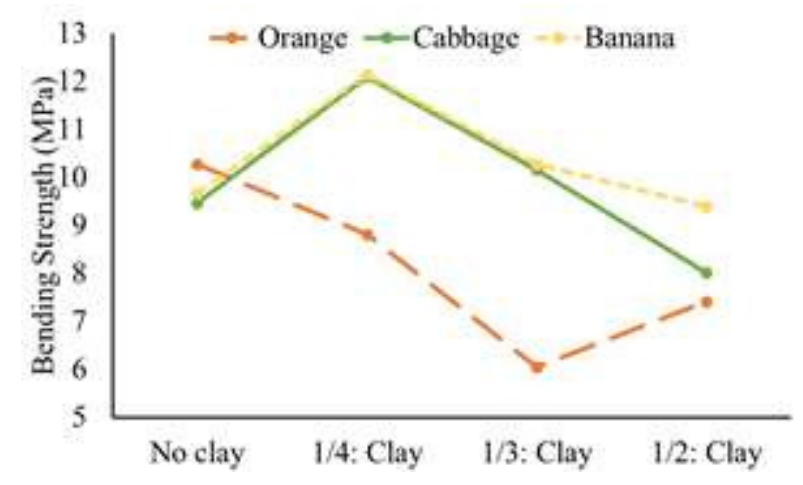

Fig. 6 Effect of edible clay proportion on bending strength

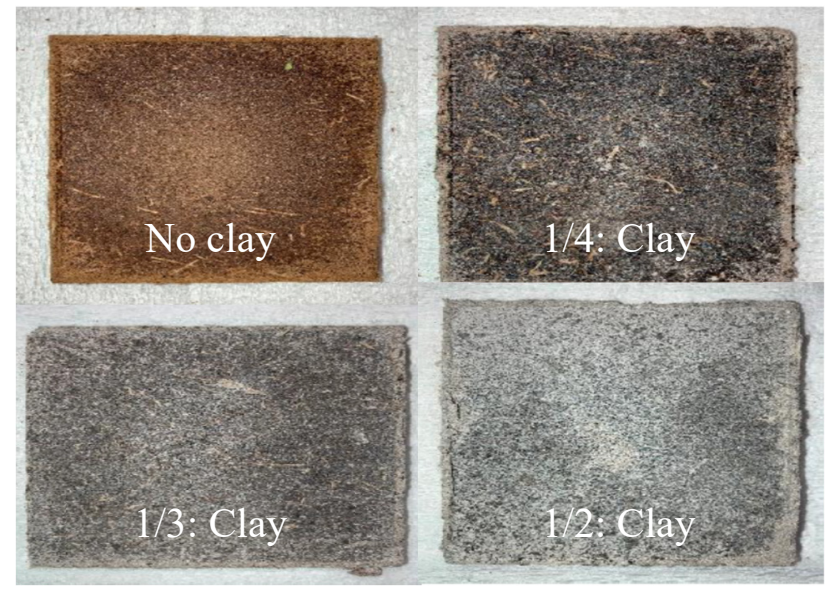

Fig. 7 Color change of banana with clay proportion

\subsection{Long-term exposure test}

Specimens exposed to air in a room lost almost all their smell in comparison with sealed specimens in four months. However, the appearances of the exposed specimens remained the same as those of the specimens before exposure. No damage to the surface or specimens such as rot, insects, worms, or fungi was observed. 
This is a transaction of the proceedings of The 70th Annual Meeting of The Society of Materials Science, Japan as "Development of Novel Construction Material from Food Waste".

\section{Conclusion}

This study aimed to produce edible construction materials with acceptable bending strength from food waste and seaweed. The following conclusions were obtained from the experiments:

- Specimens prepared with vacuum-dried food waste had a bending strength of more than $5 \mathrm{MPa}$.

- Food waste with less bending strength could be reinforced by stronger food waste.

- Improvement of the flavors of the specimens by the addition of seasonings without damaging the bending strength was confirmed.

- The color on the surface of the specimens changed after the addition of natural edible clay.

- No rot, insects, worms, or fungi were observed after four months of exposure to air in a room.

\section{References}

1) Ministry of Agriculture, Forestry and Fisheries. (2017). Reducing Food Loss and Waste \& Promoting Recycling.

2) Masatoshi SATO. (2012). Binderless Board. The Adhesion Society of Japan Journal, Volume 48, Issue 11, Page 413-420. 